\title{
Regenerative braking in high speed railway applications: analysis by different simulation tools
}

\author{
Massimo Ceraolo, Giovanni Lutzemberger \\ DESTEC \\ University of Pisa \\ Pisa, Italy \\ lutzemberger@dsea.unipi.it
}

\author{
Amedeo Frilli, Luca Pugi \\ DIEF \\ University of Florence \\ Florence, Italy \\ luca.pugi@unifi.it
}

\begin{abstract}
Technical investigations continuously evaluate the possibility to recover significant amount of braking energy. The application normally involve conventional tramways, characterized by several stops in short-range routes. However, this possibility is becoming more and more attractive also when high-speed trains are considered. In fact, braking energy can be recovered during entry in the station, and delivered later to other trains engaged in starting acceleration. In this work, authors propose an approach based on simplified models performed with object-oriented tools as Modelica or Matlab-Simscape, which easily introduce several advantages in modelling and simulation techniques. The approach has been applied to the preliminary evaluation of one energy storage system installed along a DC line, considering a high-speed train with distributed traction power as the new Italian ETR 1000.
\end{abstract}

Keywords—high-speed train; modelling; regenerative braking; simulation; storage

\section{INTRODUCTION}

With growing diffusion of trains with distributed traction systems, the percentage of energy that can be recovered during braking is drastically increasing.

In this way, a relevant part of the kinetic energy of the train is converted in electric power, and not dissipated over the friction surfaces of the pneumatic brakes, with an immediate advantage in terms of maintenance costs.

In particular, reduced wear and prolonged life of brake pads and discs also produce benefits in terms of pollution since the wear produce solid particles and contaminants whose impact on the surrounding environment is still the matter of monitoring and research [1][2][3].

The application of regenerative braking involves the availability of a load or a storage able to manage the energy recovered from the braking of the train. The peak power to be managed during regenerative braking is roughly proportional to train speed, mass and imposed deceleration.

On tramways and light urban railways, traveling speed and equivalent inertia of the train are smaller respect to high-speed train, but the frequency of braking phases is much higher. For this reason, power management of regenerative braking is typically easier and more convenient on tramways, metro and light urban railways respect to high-speed lines. Additionally, the length of metro and tramway lines is typically lower respect to conventional lines, making easier the implementation of customized or innovative solutions. For these reasons application of energy storage systems on metro, tramways and more generally on light rail systems have been widely investigated by different authors [4][5][6].

On the other hand, travelling speed and equivalent inertia are much higher in case of high-speed trains, thus increasing the amount of kinetic energy that can be potentially recovered. However, reduced number of braking phases and length of railway lines recommend to install this stationary storage capability only in correspondence of the entry inside station, to recover the braking energy and allowing to deliver that energy later, to the other high-speed trains during their starting acceleration.

In this work, the authors have developed two simulation tools based on object-oriented programming technique as Modelica and Matlab-Simscape, which easily introduce several advantages in comparison to conventional standard tools.

The aim was to evaluate pros and cons of these objectoriented tools on an existing case study, related to a high-speed railways application. In particular, the effects of the installation of one storage system on the feeding DC network, mainly in terms of energy saving from the electrical feeding substations (ESSs), have been analysed.

\section{THE SIMULATION MODELS}

Modelling and simulation of railway systems typically require a simulation tool able to simulate the network equations, the vehicle dynamic equations, i.e. the driver, and different running phases.

A wide variety of different simulation scenarios needs to be simulated, and a high customization is required to adapt models to different kind of railway vehicles, line design and operating conditions. Models should adopt parametric approach and 
customized blocks have to be easily assembled also by users with different level of skills.

For these reason the usage of high level language and development tools is quite mandatory and often proposed in recent works. In particular the authors in their recent works, adopted a modular architecture based on Matlab-Simulink [7], [8] which are "de facto" standard for the simulation of dynamical systems and the prototyping of applications involving control, diagnostic and signal processing. One of the limits of a pure Simulink architecture is represented by some compromises that have to be introduced to preserve code modularity respect to numerical efficiency. In particular, redundant integrated state are often added to reduce algebraic loop or solve implicit algebraic relationship.

More recent development languages/tool like Modelica [9] or Matlab-Simscape environments allow the user to describe the response of a component in terms of balance equations following an approach which clearly resemble the bond graph one. Main advantages of this approach, useful for railroad system simulations, have been here highlighted:

Object-oriented and graphical development. To build complex system models by direct usage of equations is very complex and time consuming. Modelica and Matlab-Simscape allows assembly of already tested models by graphically connecting them by means of their interfaces.

Cyber-physical simulation. Modelica and Matlab-Simscape allow simulation of the so-called cyber-physical systems, in which physical hardware and its control software coexist. For instance, the physical equations of the electric drive can be con be combined with the equation of the driver model, as described in [4].

Hybrid simulation. Sometimes the equations need to be changed at some times. One typical example is the structure of the feeding system that changes over time with the train position [4]. This approach creates many numerical problems if not properly addressed. This is treated in a sound way, since the simulation is a hybrid combination of continuous simulation and discrete-time events. Discrete equations are instant equations, which define the commutation of a system from a configuration to another, in a numerically sound way.

However, Modelica is much more powerful, since it is not just a commercial tool, but also a program language. In fact, it is an open environment. The models created using this language can be used within a wealth of simulating tools, either commercial or free and open-source.

\section{RESULTS ON THE APPLICATION}

\section{A. The considered case study}

The ETR 1000 is the last high speed train developed for Trenitalia by Bombardier and AnsaldoBreda.

The train whose main features are listed in Table 1, has a traction system whose corresponding performance is described in Fig. 1. The Train is designed to operate under different electrification standards in order to be potentially able to respect interoperability standards of the most important railway network of Europe.

TABLE I. CHARACTERISTICS OF THE ETR1000

\begin{tabular}{|l|c|}
\hline Total Seats & About 470 \\
\hline $\begin{array}{l}\text { Car-body } \\
\text { Construction }\end{array}$ & $\begin{array}{c}\text { Aluminium } \\
\text { Alloy }\end{array}$ \\
\hline Train Mass $(\mathrm{t})$ & 500 \\
\hline Train length $(\mathrm{m})$ & 202 \\
\hline Axle Load $(\mathrm{t})$ & 17 \\
\hline Gauge $(\mathrm{mm})$ & 1435 \\
\hline Wheel diameter $(\mathrm{mm})$ & 920 \\
\hline Nominal Power $(\mathrm{MW})$ & 9.8 \\
\hline Max Tractive Effort $(\mathrm{kN})$ & 370 \\
\hline Max Speed $(\mathrm{km} / \mathrm{h})$ & 400 \\
\hline
\end{tabular}

In this work, it was considered the operation under $3 \mathrm{kV} \mathrm{DC}$ catenary of the line Firenze-Roma that is the oldest and perhaps more important high-speed line of the Italian network. In Table 2, main electrical features of the considered line are shown.

$\begin{aligned} & \text { TABLE II. MAIN CHARACTERISTICS } \\
& \text { OF HIGH SPEED LINE "DIRETTISSIMA" }\end{aligned}$
\begin{tabular}{|l|c|}
\hline Line Impedance $(\Omega / \mathrm{km})$ & 0.05 \\
\hline ESS No Load Voltage $(\mathrm{V})$ & 3700 \\
\hline ESS Eq. Impedance $(\Omega)$ & 0.09 \\
\hline Mean Distance between SSE $(\mathrm{km})$ & 14.7 \\
\hline Min Distance between SSE $(\mathrm{km})$ & 12 \\
\hline Max Distance between SSE $(\mathrm{km})$ & 16.8 \\
\hline
\end{tabular}

The traction system of ETR 1000 is able to implement regenerative braking, however energy recovery is reduced by the availability of a load along the line since the ESSs, and the Power Stations currently installed are designed to operate in the first quadrant. In order to protect the line from potentially harmful overvoltage, regenerative braking of the train is turned off, switching to dissipative one if the line overvoltage exceed a value of about $3900 \mathrm{~V}$.

In this work, the authors investigate feasibility and potential advantages of the application of energy storages technologies in order to exploit the regenerative braking capabilities of the train.

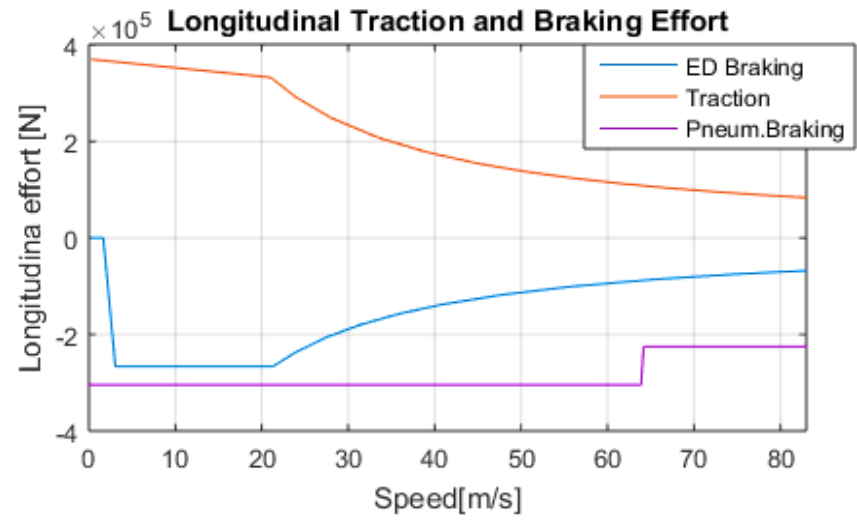

Fig. 1. Traction and Braking Performances of ETR 1000 HS Train 


\section{B. Simulation results}

The first model under analysis has been realised in MatlabSimscape. Since ETR 1000 is a relatively new project, there was a poor availability in terms of experimental data for the model validation.

Indeed, the authors use some reference experimental data [8] concerning a traction maneuver of a train on the high-speed line Roma-Firenze from 0 to $250 \mathrm{~km} / \mathrm{h}$. The maneuver has been performed applying the maximum traction effort. Comparing simulated and measured speed profile, it was possible to preliminary verify the main parameters concerning traction performance, inertia and motion resistance.

Then, electric drive measured efficiency has been matched with the simulated one. To do that, in the current model efficiency depends only from the traveling speed of the train. Once efficiency of the traction system has been roughly estimated, it was possible to refine the parameters concerning the position of ESSs of the line, starting from voltage measurements at the pantograph.

The correspondence between simulation results respect to experimental data is acceptable, considering the simplicity of the adopted model, tolerance on several parameters and availability of experimental data.

After validating the model realised in Matlab-Simscape, simulations have been performed with the other tool, based on Modelica language. Some physical and control parameters have been modified, in order to obtain identical results. Indeed, a mutual validation between the two programming techniques has been performed, as shown in Fig. 2.
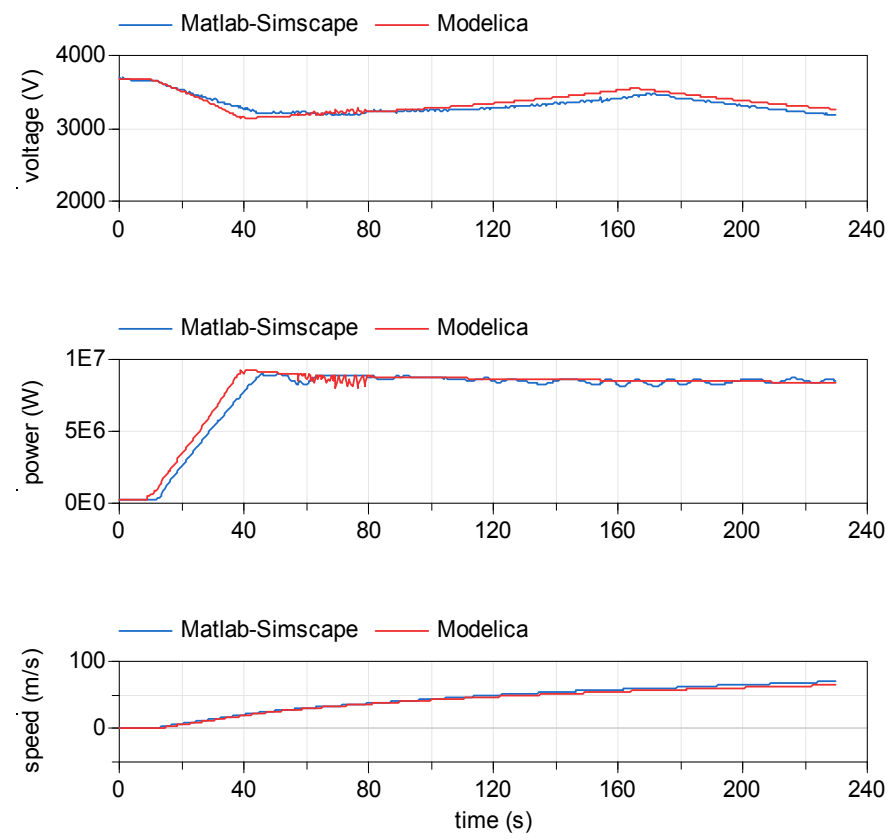

Fig. 2. Results under the considered traction speed profile

As visible, simulation outputs are nearly equivalent, substantially in agreement with the experimental data.
It must however be said that realization of the tool based on Modelica language has allowed a transfer of knowledge from the commercial tool to one open environment, improving flexibility and development potential, with clear and evident benefits for the user.

\section{Braking energy evaluation}

After mutually validating the two models on the same traction maneuver, the attention has been posed on one braking maneuver, to evaluate the amount of recoverable energy, in correspondence to one terminal. In particular, entrance in the rail junction of Florence was chosen. In this regard, following assumptions have been made:

- The braking speed profile is considered perfectly symmetric respect to the acceleration phase, already shown in the bottom part of Fig. 2.

- Efficiency map of the electric drive during braking has been considered the same as in traction.

- Mutual distance between ESSs in correspondence of the terminal was extrapolated starting from those located in the middle part of the track, already used for validation.

To enhance energy recovery during braking, otherwise constrained by the presence of other trains that at the same time are adsorbing power in the vicinity of itself, the installation of one storage system in correspondence of the last ESS (i.e. the ESS nearer to the terminal) was taken into account. This was effectively simulated by including a battery model of the type already shown in [10].

\section{Storage system sizing}

The selected storage based with high power lithium batteries has been sized accordingly to the hypothesis to consider only the trains travelling on the DC high-speed railway, independently by the rest of the traffic on the rail junction. Indeed, the energy recovered by one train entering in the station can be effectively reused by another train, placed on the same DC high-speed railway line.

This can be physically allowed by the presence of a DC/DC converter between the storage and the grid, of the same typology already considered in [5], to activate the energy recovery inside the storage system only when trains along the DC high-speed railway line are present.

At first step, sizing of the storage has been performed without considering the presence of the DC/DC converter. In this regard, the number of cells has been chosen in such a ways that when the contact line is at its nominal voltage, the SOC stays at an intermediate level, and the nominal capacity is selected according to the maximum allowed current stress.

Additionally, the selected capacity has been corrected also in consideration of the cell life expectation. In this regard, a rough estimation of 25 trains per day entering/going out in the railway junction can be considered. Indeed, under the hypothesis of a global duration for the application of around 10 years, about $10^{5}$ charging-discharging cycles can be estimated. Even though no clear evidence exists in literature, experiences made by the authors [11] and some available 
manufacturer's indications [12] show that the depth of discharge corresponding to such micro-cycles is around $10 \%$.

Finally, a solution based on NMC cells expressly dedicated for high power applications has been selected, as shown in Table III.

TABLE III. STORAGE SYSTEM CHARACTERISTICS

\begin{tabular}{|l|c|}
\hline Number of cells & 984 \\
\hline Nominal voltage (V) & 3640 \\
\hline Nominal capacity (Ah) & 200 \\
\hline Nominal energy (kWh) & 728 \\
\hline
\end{tabular}

Regarding the energy saving from ESSs, it can be easily determined by evaluating the energy recovered inside the storage system during braking. In fact, this energy can be assigned to another train that is going out, thus consequently reducing the delivered energy by the ESS nearer to the terminal. Naturally, charging-discharging efficiency from the storage must be taken into account.

As noticeable from Fig. 3, during braking (first plot from the top) voltage rises up the maximum admitted voltage (second plot from the top), and some of the recoverable energy is dissipated on resistors on board trains. The other part is recovered inside the storage. Always considering a number of 25 trains per day and a recovered energy around $350 \mathrm{MJ}$ (third plot from the top) for single braking, including a chargingdischarging efficiency of 0.9 , it is possible to estimate an annual energy saving from ESSs equal to $797 \mathrm{MWh}$. It can be observed, according to the storage sizing criteria previously mentioned, that maximum $\triangle \mathrm{SOC}$ is under $10 \%$ (fourth plot from the top) according to the considered cell life expectation.
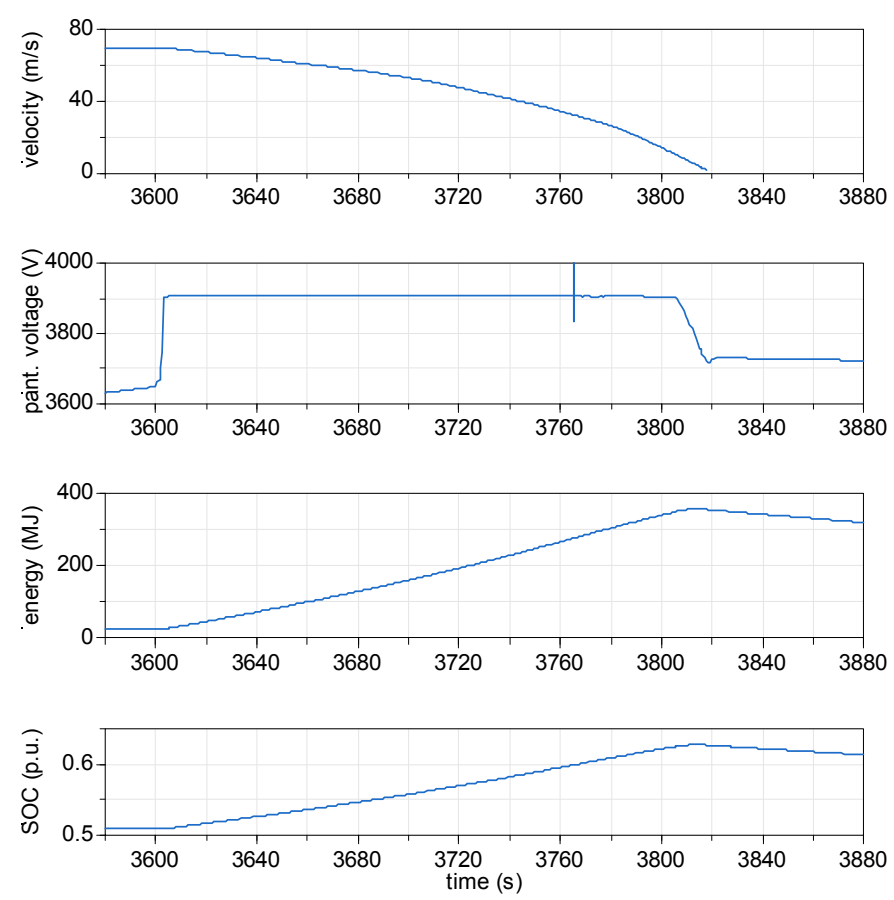

Fig. 3. Results under the considered braking speed profile

\section{E. Cost analysis}

In the previous section, it was shown that the addition of a single storage system to the line involves, for the considered case-study, an annual reduction in the total energy bought from the mains of $797 \mathrm{MWh} / \mathrm{y}$. The quantification of the corresponding money advantage depends on the particular contract for the provision of energy. However, considering the current industrial user prices of energy in Italy (i.e. an average value of $100 € / \mathrm{MWh}$ ) the annual savings due to the installation of the storage system is estimated in around $80 \mathrm{k} € / \mathrm{y}$.

As regards the storage system cost, considering the high power cells needed, a cost of $600 € / \mathrm{kWh}$ is reasonable, also including the case, connections, Battery monitoring System (BMS), electromechanical protection. All this considered, the capital expenditure for the whole storage system which characteristics shown in Table III is evaluated to be around $440 \mathrm{k} €$.

The numbers are so simple that it is not necessary to make the full cash-flow computations to conclude that the payback time of the proposed system is around five years that combined with the expected life of ten years makes it attractive.

\section{CONCLUSIONS}

The paper has shown that the addition of energy recovering capability to high-speed railway lines can bring significant amount of energy saving when one stationary storage system is installed in correspondence of one ESS located in correspondence of the entrance in a railway junction.

Among the several possible alternatives, the paper has focused on the option of installing a single storage system based with high power lithium cells, without interposing between the storage and the line any DC/DC converter.

In a realistic case study, involving a $728 \mathrm{kWh}$ storage and only considering trains moving on the DC high-speed line, payback time is expected around five years, for a system that is expected to stay in service ten years.

Further advantages could be obtained by including recoverable energy also by trains travelling on other railway lines, entering in the same railway junction.

\section{REFERENCES}

[1] S. Abbasi, A. Jansson, L. Olander, U. Olofsson, U. Sellgren, "A pin-ondisc study of the rate of airborne wear particle emissions from railway braking materials", Wear, 284, pp.18-29.

[2] R. Gehrig, M. Hill, P. Lienemann et alii, "Contribution of railway traffic to local PM10 concentrations in Switzerland", Atmospheric Environment, 41 (5), pp.923-933.

[3] I. Salma, T. Weidinger, W. Maenhaut, "Time-resolved mass concentration, composition and sources of aerosol particles in a metropolitan underground railway station", Atmospheric Environment, 41 (37).

[4] M. Ceraolo, M. Conte, R. Giglioli, G. Lutzemberger, M. Pasquali: "Use of electrochemical storage in substations to enhance energy and cost efficiency of tramways", AEIT Annual Conference, Oct. 2013, Mondello.

[5] M. Ceraolo, G. Lutzemberger, "Stationary and on-board storage systems to enhance energy and cost efficiency of tramways", Journal of Power Sources, vol. 264, 2014, pp. 128-139.

[6] R. Barrero, X. Tackoen, J. van Mierlo, "Stationary or onboard energy storage systems for energy consumption reduction in a metro network", 
Proceedings of the Institution of Mechanical Engineers, Part F: Journal of Rail and Rapid Transit, vol. 224, pp. 207-225, 2010.

[7] R. Conti, E. Galardi, E. Meli, D. Nocciolini, L. Pugi, A. Rindi, "Energy and wear optimisation of train longitudinal dynamics and of traction and braking systems", Vehicle System Dynamics, 53 (5), pp. 651-671, 2015.

[8] A. Frilli, E. Meli, D. Nocciolini, L. Pugi, A. Rindi, "Object Oriented Simulation of Longitudinal Train Dynamics Efficient Tools to Optimize Sustainability and Efficiency of Railway Systems", Proceedings of 2015 AEIT International Annual Conference. p. 1-6, AEIT, Napoli, 14-16 Ottobre 2015.
[9] P. Fritzson, "Introduction to Modeling and Simulation of Technical and Physical Systems with Modelica", Wiley-IEEE Press, 2011.

[10]M. Ceraolo, T. Huria, G. Lutzemberger, "Experimentally-determined models for high-power lithium batteries", SAE 2011 World Congress \& Exhibition, SAE Technical Paper, 2011-01-1365.

[11]M. Ceraolo, G. Lutzemberger, M. Marracci, High power Lithium Batteries usage in hybrid vehicles, Vehicle Power and Propulsion Conference (VPPC), 1-3 Sept. 2010.

[12]Kokam official site, http://www.kokam.com, last access: 28/12/2015. 